\title{
Outcome for fetuses with a diagnosis of myelomeningocele, Hydrocephalus and Brain Anomalies Daniel Doherty ${ }^{2}$, David Shurtleff², Sharon Duguay ${ }^{1}$ and Anthony Avellino*2
}

\author{
Address: ${ }^{1}$ Department of Neurosurgery, University of Washington, Seattle, WA, 98195, USA and ${ }^{2}$ Department of Pediatrics, University of \\ Washington, Seattle, WA, 98195, USA \\ Email: Anthony Avellino* - anthony.avellino@seattlechildrens.org \\ * Corresponding author
}

from 50th Annual Meeting of the Society for Research into Hydrocephalus and Spina Bifida

Cambridge, UK. 30 August - 2 September 2006

Published: 21 December 2006

Cerebrospinal Fluid Research 2006, 3(SuppI I):S22 doi:I0.II86/I743-8454-3-SI-S22

(C) 2006 Doherty et al; licensee BioMed Central Ltd.

\section{Background}

Since the early 1980 's foetal malformations can be diagnosed by increasingly sophisticated ultrasound (US) examinations. Long term developmental follow up has not been reported.

\section{Materials and methods}

We will report the outcome for all 241 patients referred over the past 25 years by tertiary level Obstetricians to our Neurodevelopmental team to either explain the options for treatment of their affected foetus or to provide prognosis for their determination to terminate the pregnancy or carry to term. They include all patients in the cohort and include the following diagnoses: Major brain malformations - 22, Hydrocephalus - 45, Syndromes -3 and Myelomeningocele $(\mathrm{MM})-171$. All but one case with MM were delivered by caesarean section birth before the rupture of amniotic membranes. Analysis was by $\mathrm{X}^{2}$ or Fishers Exact.

\section{Results}

We averaged 7 to 8 consults per year with a range of 1 to 18. The fetal ages ranged from 17 to 36 weeks. Foetuses with MM 24 weeks gestational age (EGA) or older were terminated less frequently 4 of $74(4 \%)$ than those of less than 24 weeks EGA, 8 of 41 (19.5\%), $\mathrm{P}=0.015$. Termination rates for the same EGA groups with hydrocephalus were $0 \%$ and $17 \%$, an insignificant difference. Of the foetuses with MM given a "unfavorable" prognosis - high rate of mental retardation and "severe" disability, 21\% were terminated and $17 \%$ of those assessed as having sufficient intellectual ability to become self sufficient, $\mathrm{P}=0.5$. For all foetuses with MM the outcomes were: terminations $20 \%$; still born $3 \%$; severe Chiari II symptoms $2 \%$; postnatal motor level 2 levels higher than predicted from in utero estimates $5 \%$, 1 level $10 \%$, equal $32 \%$, and lower by 1 level 14\%, 2 levels $9 \%$ and 3 levels $5 \%$ and $?=25 \%$. Sensory levels were assessed postnally compared to the patients' motor level at the same time as follows; sensory level was higher by 3 levels $=2 \%, 2$ levels $=17 \%, 1$ level $=$ $42 \%$ and equal only in 39\% with none lower than the motor level. Functional level achieved by those old enough to evaluate were as follows, Preschool and Kindergarten $\mathrm{L} 3$ and above $36 \%$ with $\mathrm{L} 4$ and below $=13 \%$; grade school $=28 \%$ and $52 \%$, normal $=20 \%$ and $22 \%$, mentally deficient $16 \%$ and $13 \%$ respectively, $\mathrm{P}=0.12$. The $\mathrm{L} 3$ and above group had $16 \%$ moderate and severely mentally retarded (MR) and the L4 and below had mild and moderate $\mathrm{MR}=13 \%$. For those foetuses with MM who survived to be older than 8 years of age, 11 of $15(73 \%)$ with a favourable prognosis were developing normally and 13 of 22 with an unfavourable prognosis, $\mathrm{P}=0.7$. The small numbers in the hydrocephalus and brain anomalies groups prevent statistical analysis.

\section{Conclusion}

Some parents in the USA carry the pregnancy to term despite severe fetal diagnoses. We lack data about long term outcome of anomalies with poor prognoses. 